\title{
Precision Engine for Nanobiomedical Research
}

\section{Afonin SM}

National Research University of Electronic Technology, MIET, Moscow, Russia.

Corresponding Author: Afonin Sergey Mikhailovich, National Research University of Electronic Technology, MIET, 124498, Moscow, Russia.

Received Date: February 08, 2021; Accepted Date: March 01, 2021; Published Date: March 12, 2021

Citation:Afonin SM. (2021) Precision engine for nanobiomedical research. Biomedical Research and Clinical Reviews. 3(4); DOI: 10.31579/26929406/051

Copyright: (C) 2021 Afonin SM, This is an open-access article distributed under the terms of the Creative Commons Attribution License, which permits unrestricted use, distribution, and reproduction in any medium, provided the original author and source are credited.

\section{Abstract:}

The transfer function and the transfer coefficient of a precision electromagnetoelastic engine for nanobiomedical research are obtained. The structural diagram of an electromagnetoelastic engine has a difference in the visibility of energy conversion from Cady and Mason electrical equivalent circuits of a piezo vibrator. The structural diagram of an electromagnetoelastic engine is founded. The structural diagram of the piezo engine for nanobiomedical research is written. The transfer functions of the piezo engine or are obtained.

Keywords: precision engine; electromagnetoelastic engine; transfer function; structural model and diagram; nanobiomedical research; piezo engine; deformation; transfer coefficient

\section{Introduction}

A precision electromagnetoelastic engines in the form of piezo engines or magnetostriction engines are applied in nanomanipulators, nanopumps, scanning microscopes for nanobiomedical research [1-6]. The piezo engine is used for nanodisplacements in photolithography, medical equipment of microsurgical operations, adaptive optics systems and fiberoptic systems for transmitting and receiving information [4-12].

The electromagnetoelasticity equation and the differential equation are solved to construct the structural diagram of an electromagnetoelastic engine. The structural diagram of the engine has a difference in the visibility of energy conversion for from Cady and Mason electrical equivalent circuits of a piezo vibrator [4-8].

\section{Transfer function}

The structural diagram of a precision engine for nanobiomedical research is changed from Cady and Mason electrical equivalent circuits [4-8]. For a precision engine the equation of electromagnetoelasticity [2-14] has the form of the equation of the reverse effect

$$
S_{i}=d_{m i} \Psi_{m}+s_{i j}^{\Psi} T_{j}
$$

where $S_{i}, d_{m i}, \Psi_{m}, s_{i j}^{\Psi}$ and $T_{j}$ are the relative deformation, the module, the control parameter or the intensity of field, the elastic compliance, the mechanical intensity; $i=1,2, \ldots, 6 ; m=1,2,3$; and $j=1,2, \ldots, 6$ are the indexes.

The equation of the force on the face of a precision engine has the form [10-19]

$$
M d^{2} \xi(x, t) / d t^{2}+F=S_{0} T
$$

Where $M, F, \xi, x, t, S_{0}$ are the mass and force of load, the displacemen, the coordinate, the time, the area of engine.

The differential equation of a precision engine has the form [4-32]

$$
\begin{gathered}
d^{2} \Xi(x, p) / d x^{2}-\gamma^{2} \Xi(x, p)=0 \\
\gamma=p / c^{\Psi}+\alpha
\end{gathered}
$$

Where $\Xi(x, p)$ is the transform of Laplace for displacement; $p, \gamma$, $c^{\Psi}, \alpha$ are the operator of transform, the coefficient of wave propagation, the speed of sound, the coefficien of attenuation. The system of the equations for the forces on faces of a precision engine is written [10-40]

$$
\begin{gathered}
M_{1} p^{2} \Xi_{1}(p)+F_{1}(p)=S_{0} T_{j}(0, p) \\
-M_{2} p^{2} \Xi_{2}(p)-F_{2}(p)=S_{0} T_{j}(l, p)
\end{gathered}
$$

Where $\Xi_{1}(p), \Xi_{1}(p)$ are transforms displacement of faces 1 and 2 for a precision engine.

The system of equations for the structural diagram and model of a precision engine for the distributed parameters in nanobiomedical research on Figure 1 has the form 


$$
\begin{aligned}
& \Xi_{1}(p)=\left(M_{1} p^{2}\right)^{-1} \times\left\{\begin{array}{l}
-F_{1}(p)+\left(1 / \chi_{i j}^{\Psi}\right) \\
\times\left[\begin{array}{l}
d_{m i} \Psi_{m}(p)+[\gamma / \operatorname{sh}(l \gamma)] \\
\times\left[\Xi_{2}(p)-\operatorname{ch}(l \gamma) \Xi_{1}(p)\right]
\end{array}\right]
\end{array}\right\} \\
& \Xi_{2}(p)=\left(M_{2} p^{2}\right)^{-1} \times\left\{\begin{array}{l}
-F_{2}(p)+\left(1 / \chi_{i j}^{\Psi}\right) \times \\
\times\left[\begin{array}{l}
d_{m i} \Psi_{m}(p)+[\gamma / \operatorname{sh}(l \gamma)] \\
\times\left[\Xi_{1}(p)-\operatorname{ch}(l \gamma) \Xi_{2}(p)\right]
\end{array}\right]
\end{array}\right\}
\end{aligned}
$$

Where $\chi_{i j}^{\Psi}=s_{i j}^{\Psi} / S_{0}, d_{m i}=\left\{\begin{array}{l}d_{33}, d_{31}, d_{15} \\ d_{33}, d_{31}, d_{15}\end{array}, \Psi_{m}=\left\{\begin{array}{l}E_{3}, E_{1} \\ H_{3}, H_{1}\end{array}\right.\right.$, $s_{i j}^{\Psi}=\left\{\begin{array}{c}s_{33}^{E}, s_{11}^{E}, s_{55}^{E} \\ s_{33}^{H}, s_{11}^{H}, s_{55}^{H}\end{array}, \gamma=\left\{\begin{array}{c}\gamma^{E} \\ \gamma^{H}\end{array}, E\right.\right.$ is the intensity of electric field, $H$ is the intensity of magnetic field.

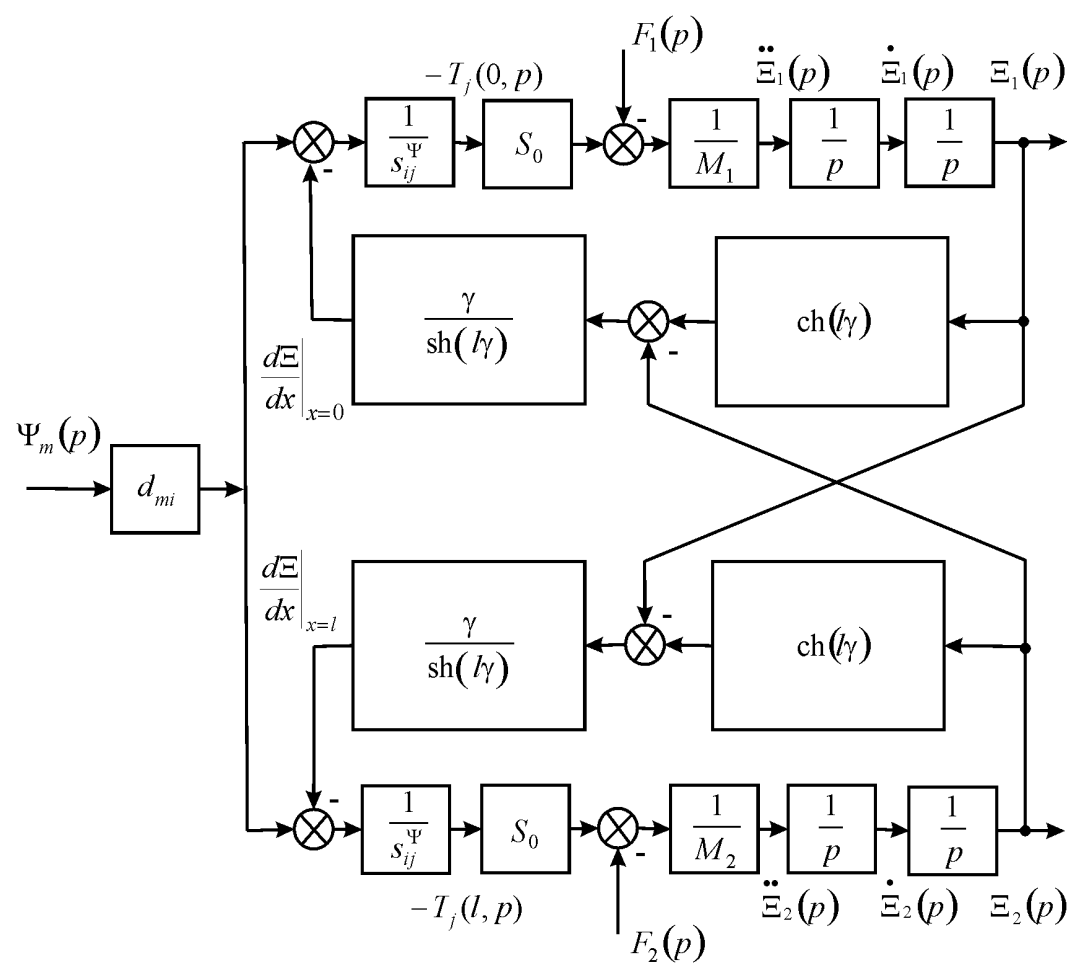

Figure 1: Structural diagram of precision engine for distributed parameters.

The matrix equation of a precision engine for nanobiomedical research has the form

$$
\left(\begin{array}{l}
\Xi_{1}(p) \\
\Xi_{2}(p)
\end{array}\right)=\left(\begin{array}{lll}
W_{11}(p) & W_{12}(p) & W_{13}(p) \\
W_{21}(p) & W_{22}(p) & W_{23}(p)
\end{array}\right)\left(\begin{array}{c}
\Psi_{m}(p) \\
F_{1}(p) \\
F_{2}(p)
\end{array}\right)
$$

The equation of the direct piezoelectric effect for the piezo engine in nanobiomedical research [10-14] has the form

$$
D_{m}=d_{m i} T_{i}+\varepsilon_{m k}^{E} E_{k}
$$

Where $D_{m}, \varepsilon_{m k}^{E}$ are the electric induction and the permittivity; $k=1,2,3$ is the index.

The equation for the coefficient of the direct piezoelectric effect $k_{d}$ for the piezo engine at $E=$ const has the form

$$
k_{d}=\frac{I_{\dot{\Xi}_{n}}(p)}{\dot{\Xi}_{n}(p)}=\frac{d_{m i} S_{0}}{\delta s_{i j}^{E}}, \quad n=1,2
$$

Where $I_{\dot{\Xi}_{n}}(p), \dot{\Xi}_{n}(p)$ are transforms of current and velocity; $n$ is number of the face engine.

After conversion the structural diagram on Figure 1 the structural diagram of the piezo engine for nanobiomedical research has form Figure 2. The equation for negative feedback for structural diagram of piezo engine on Figure 2 has the form

$$
U_{\dot{\Xi}_{n}}(p)=\frac{d_{m i} S_{0} R}{\delta s_{i j}^{E}} \dot{\Xi}_{n}(p), \quad n=1,2
$$

The equation for $k_{r}$ the coefficient of the reverse piezoelectric effect for the piezo engine in nanobiomedical research is obtained in the form

$$
k_{r}=k_{d}=\frac{d_{m i} S_{0}}{\delta s_{i j}^{E}}
$$




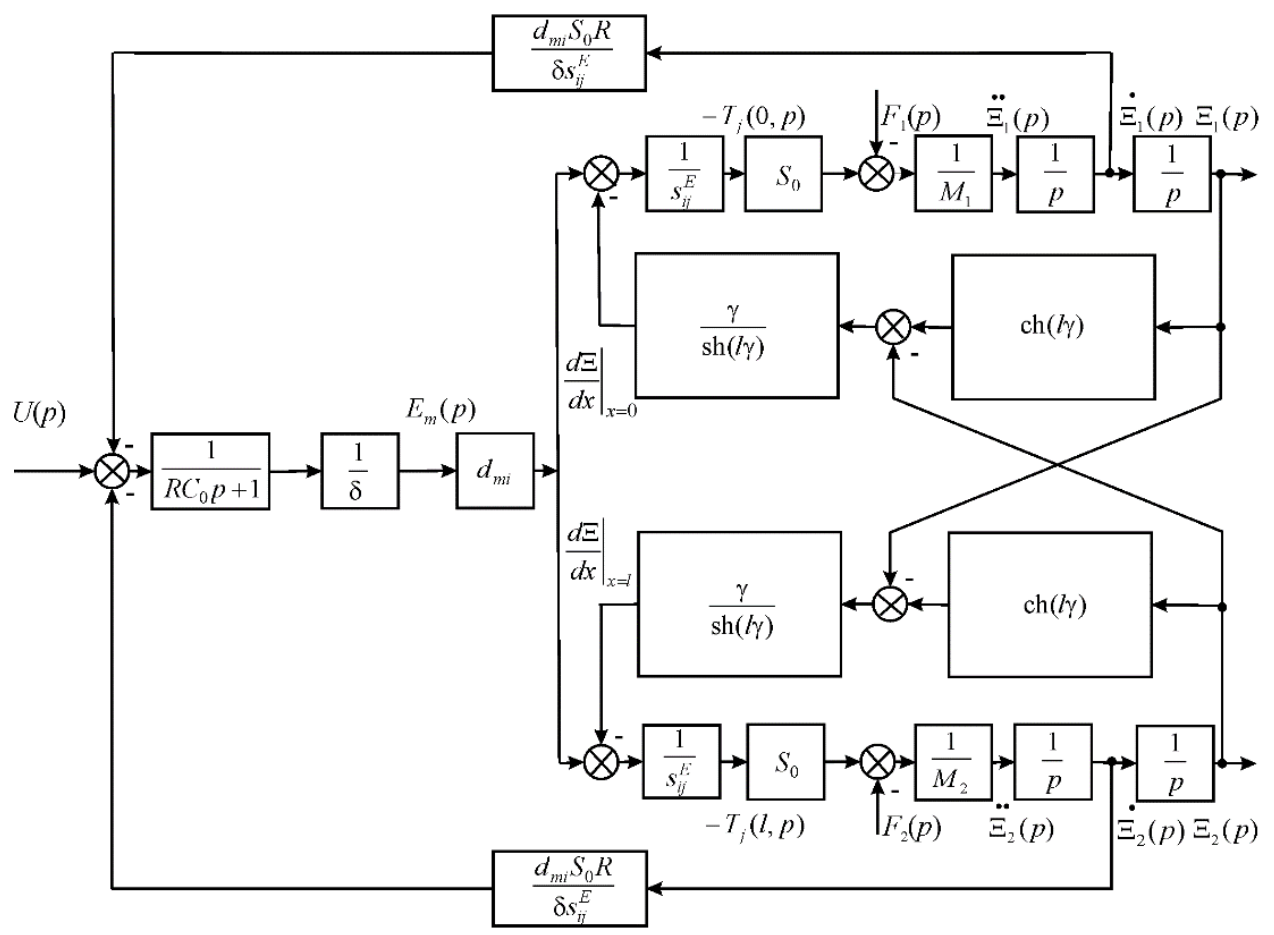

Figure 2: Structural diagram of piezo engine for nanobiomedical research.

The structural diagram of the piezo engine with one fixed face for the lumped parameters is received on Figure 3.

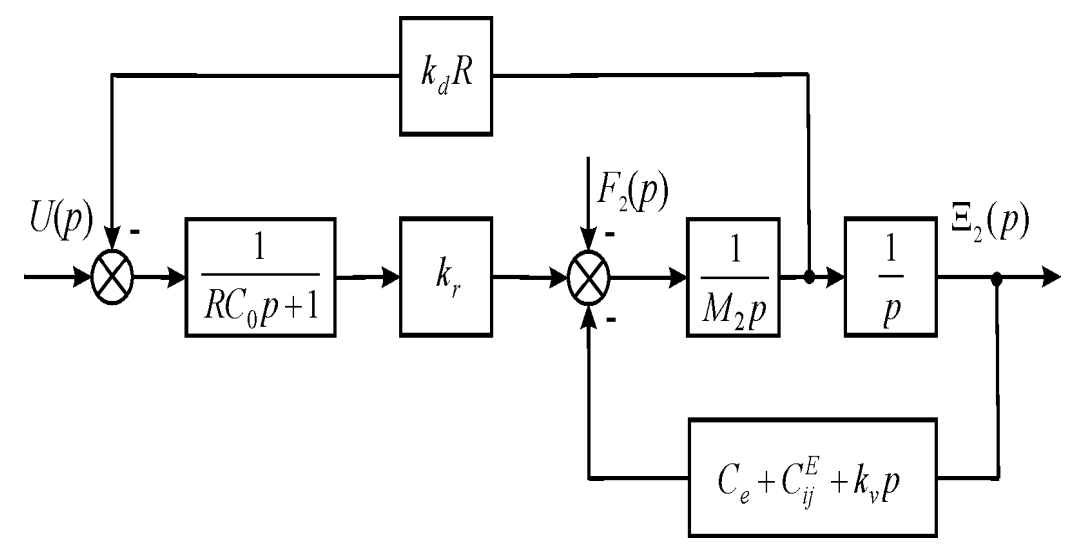

\section{Figure 3: Structural diagram of piezo engine for lumped parameters.}

The transfer function of the piezo engine for the lumped parameters in nanobiomedical research on Figure 3 at $R=0$ has the form

$$
W(p)=\frac{\Xi_{2}(p)}{U(p)}=\frac{k_{r}}{M_{2} p^{2}+k_{v} p+C_{i j}^{E}+C_{e}}
$$

Where $U(p)$ is transformations of the voltage. Therefore, the transfer function of the piezo engine has the follow form

$$
W(p)=\frac{\Xi_{2}(p)}{U(p)}=\frac{k_{m i}^{U}}{T_{t}^{2} p^{2}+2 T_{t} \xi_{t} p+1}
$$

$$
k_{m i}^{U}=\left(d_{m i} l / \delta\right) /\left(1+C_{e} / C_{i j}^{E}\right), \quad T_{t}=\sqrt{M_{2} /\left(C_{i j}^{E}+C_{e}\right)}
$$

Where $k_{m i}^{U}, T_{t}, \xi_{t}$ are the transfer coefficient, the time constant, the coefficient attenuation; $C_{i j}^{E}=S_{0} /\left(s_{i j}^{E} l\right)=1 /\left(\chi_{i j}^{E} l\right)$ is the stiffness of the piezo engine at $\mathrm{E}=$ const. The transfer function of the transverse piezo engine has the form

$$
\begin{gathered}
W(p)=\frac{\Xi_{2}(p)}{U(p)}=\frac{k_{31}^{U}}{T_{t}^{2} p^{2}+2 T_{t} \xi_{t} p+1} \\
k_{31}^{U}=\left(d_{31} h / \delta\right) /\left(1+C_{e} / C_{11}^{E}\right), \quad T_{t}=\sqrt{M_{2} /\left(C_{e}+C_{11}^{E}\right)}
\end{gathered}
$$


Where $h, \delta$ are the height and the thickness; $C_{11}^{E}=S_{0} /\left(s_{11}^{E} h\right)=1 /\left(\chi_{11}^{E} h\right)$ is the stiffness of the transverse piezo engine at $E=$ const .

The transient process of the piezo engine at the transverse piezoelectric effect at the step input voltage has the form

$$
\begin{gathered}
\xi_{2}(t)=k_{31}^{U} U\left(1-\frac{e^{-\frac{\xi_{t} t}{T_{t}}}}{\sqrt{1-\xi_{t}^{2}}} \sin \left(\omega_{t} t+\varphi_{t}\right)\right. \\
\omega_{t}=\frac{\sqrt{1-\xi_{t}^{2}}}{T_{t}}, \quad \varphi_{t}=\operatorname{arctg}\left(\frac{\sqrt{1-\xi_{t}^{2}}}{\xi_{t}}\right)
\end{gathered}
$$

For the piezo engine with the transverse piezoelectric effect made from ceramic PZT at $d_{31}=2 \cdot 10^{-10} \mathrm{~m} / \mathrm{V}, h / \delta=16, M_{2}=2 \mathrm{~kg}, C_{11}^{E}=$ $2.8 \cdot 10^{7} \mathrm{~N} / \mathrm{m}, C_{e}=0.4 \cdot 10^{7} \mathrm{~N} / \mathrm{m}, U=25 \mathrm{~V}$, its parameters are obtained $T_{t}=0.25 \cdot 10^{-3} \mathrm{~s}$ and steady-state displacement $\left.\xi_{2}(t)\right|_{t \rightarrow \infty}=\xi_{2}(\infty)=\Delta h$ $=70 \mathrm{~nm}$.

The characteristics of an electromagnetoelastic engine for nanobiomedical research are obtained. The mechanical characteristic of the engine is received as $S_{i}\left(T_{j}\right)$ or $\Delta l(F)$ [10-15]

$$
\left.S_{i}\right|_{\Psi=\text { const }}=\left.d_{m i} \Psi_{m}\right|_{\Psi=\text { const }}+S_{i j}^{\Psi} T_{j}
$$

The regulation characteristics of an electromagnetoelastic engine for nanobiomedical research is obtained as $S_{i}\left(\Psi_{m}\right)$ or $\Delta l(U)$ [10-15]

$$
\left.S_{i}\right|_{T=\text { const }}=d_{m i} \Psi_{m}+\left.s_{i j}^{\Psi} T_{j}\right|_{T=\text { const }}
$$

The mechanical characteristic of a precision engine has the following form

$$
\Delta l=\Delta l_{\max }\left(1-F / F_{\max }\right)
$$

The maximum of the parameters $\Delta l_{\max }$ and $F_{\max }$ of the mechanical characteristic of a precision engine have the form

$$
\begin{gathered}
\Delta l_{\max }=d_{m i} \Psi_{m} l \\
F_{\max }=T_{j \max } S_{0}=d_{m i} \Psi_{m} S_{0} / s_{i j}^{\Psi}
\end{gathered}
$$

Where index max is used for the maximum value of parameter of a precision engine.

The maximum values of parameters of the piezo engine for the transverse piezoelectric effect have the form

$$
\Delta h_{\max }=d_{31} E_{3} h
$$

$$
F_{\max }=d_{31} E_{3} S_{0} / s_{11}^{E}
$$

For the transverse piezo engine at $d_{31}=2 \cdot 10^{-10} \mathrm{~m} / \mathrm{V}, E_{3}=0.5 \cdot 10^{5}$ $\mathrm{V} / \mathrm{m}, h=2.5 \cdot 10^{-2} \mathrm{~m}, S_{0}=1.5 \cdot 10^{-5} \mathrm{~m}^{2}, s_{11}^{E}=15 \cdot 10^{-12} \mathrm{~m}^{2} / \mathrm{N}$ its parameters on are found $\Delta h_{\max }=250 \mathrm{~nm}$ and $F_{\max }=10 \mathrm{~N}$. Theoretical and practical parameters of the piezo engines are coincidences with an error of $10 \%$.

The regulation characteristic of an electromagnetoelastic engine for nanobiomedical research at elastic load $F=C_{e} \Delta l$ is obtained in the form

$$
\frac{\Delta l}{l}=d_{m i} \Psi_{m}-\frac{s_{i j}^{\Psi} C_{e}}{S_{0}} \Delta l
$$

The equation of the displacement of an electromagnetoelastic engine at elastic load has the form

$$
\Delta l=\frac{d_{m i} l \Psi_{m}}{1+C_{e} / C_{i j}^{\Psi}}
$$

The equation of the displacement of the transverse piezo engine at elastic load has the form

$$
\Delta h=\frac{\left(d_{31} h / \delta\right) U}{1+C_{e} / C_{11}^{E}}=k_{31}^{U} U
$$

For the transverse piezo actuator at $d_{31}=2 \cdot 10^{-10} \mathrm{~m} / \mathrm{V}, h / \delta=20, C_{11}^{E}$ $=2 \cdot 10^{7} \mathrm{~N} / \mathrm{m}, C_{e}=0.2 \cdot 10^{7} \mathrm{~N} / \mathrm{m}, U=55 \mathrm{~V}$, its parameters are found $k_{31}^{U}=3.64 \mathrm{~nm} / \mathrm{V}$ and steady-state displacement $\left.\xi_{2}(t)\right|_{t \rightarrow \infty}=\xi_{2}(\infty)=\Delta h=200 \mathrm{~nm}$.

For calculations the mechatronics control systems for nanobiomedical research with a precision engine its characteristics are found.

\section{Conclusions}

The transfer function and the transfer coefficient of a precision electromagnetoelastic engine are received. The structural diagram of a precision engine for nanobiomedical research is obtained. The structural diagram of an electromagnetoelastic engine for nanobiomedical research is distinguished by the clarity of energy conversion from Cady and Mason electrical equivalent circuits of a piezo vibrator.

The electromagnetoelasticity equation and the differential equation are used to construct the structural diagram of an electromagnetoelastic engine. The structural diagram of an electromagnetoelastic engine is found from its electromagnetoelasticity and differential equations. The structural diagram of the piezo engine is received using the reverse and direct piezoelectric effects. The back electromotive force for the piezo engine is founded from the direct piezoelectric effect. The characteristics of a precision engine for nanobiomedical research are obtained.

\section{References}

1. Schultz J, Ueda J, Asada H. (2017) Cellular Actuators. Butterworth-Heinemann Publisher, Oxford, 382.

2. Afonin SM. (2006) Absolute stability conditions for a system controlling the deformation of an elecromagnetoelastic transduser. Doklady Mathematics 74(3): 943-948.

3. Uchino K (1997) Piezoelectric actuator and ultrasonic motors. 
Boston, MA: Kluwer Academic Publisher. 347.

4. Afonin SM. (2005) Generalized parametric structural model of a compound elecromagnetoelastic transduser. Doklady Physics 50(2): 77-82.

5. Afonin SM. (2008) Structural parametric model of a piezoelectric nanodisplacement transducer. Doklady Physics 53(3): 137-143.

6. Afonin SM. (2006) Solution of the wave equation for the control of an elecromagnetoelastic transduser. Doklady Mathematics 73(2): 307-313.

7. Cady WG. (1946) Piezoelectricity: An introduction to the theory and applications of electro mechancial phenomena in crystals. McGraw-Hill Book Company, New York, London, 806.

8. Physical Acoustics: Principles and Methods. Vol.1. Part A. Methods and Devices. Ed.: Mason W (1964). Academic Press, New York, 515.

9. Zwillinger D. (1989) Handbook of Differential Equations. Academic Press, Boston, 673.

10. Afonin SM. (2006) A generalized structural-parametric model of an elecromagnetoelastic converter for nano- and micrometric movement control systems: III. Transformation parametric structural circuits of an elecromagnetoelastic converter for nanoand micrometric movement control systems, Journal of Computer and Systems Sciences International 45(2): 317-325.

11. Afonin SM. (2016) Decision wave equation and block diagram of electromagnetoelastic actuator nano- and microdisplacement for communications systems. International Journal of Information and Communication Sciences 1(2): 22-29.

12. Afonin SM. (2015) Structural-parametric model and transfer functions of electroelastic actuator for nano- and microdisplacement. Chapter 9 in Piezoelectrics and Nanomaterials: Fundamentals, Developments and Applications. Ed. Parinov IA. Nova Science, New York, pp. 225-242.

13. Afonin SM. (2017) A structural-parametric model of electroelastic actuator for nano- and microdisplacement of mechatronic system. Chapter 8 in Advances in Nanotechnology. Volume 19. Eds. Bartul Z, Trenor J, Nova Science, New York, pp. 259-284.

14. Afonin SM. (2018) Electromagnetoelastic nano- and microactuators for mechatronic systems. Russian Engineering Research 38(12): 938-944.

15. Afonin SM. (2012) Nano- and micro-scale piezomotors. Russian Engineering Research 32(7-8): 519-522.

16. Afonin SM. (2007) Elastic compliances and mechanical and adjusting characteristics of composite piezoelectric transducers, Mechanics of Solids 42(1): 43-49.

17. Afonin SM. (2014) Stability of strain control systems of nano-and microdisplacement piezotransducers. Mechanics of Solids 49(2): 196-207.

18. Afonin SM. (2017) Structural-parametric model electromagnetoelastic actuator nanodisplacement for mechatronics. International Journal of Physics 5(1): 9-15.

19. Afonin SM. (2019) Structural-parametric model multilayer electromagnetoelastic actuator for nanomechatronics. International Journal of Physics 7(2): 50-57.

20. Afonin SM. (2017) Structural-parametric model of piezoactuator nano- and microdisplacement for nanoscience. AASCIT Journal of Nanoscience 3(3): 12-18.

21. Afonin SM. (2016) Solution wave equation and parametric structural schematic diagrams of electromagnetoelastic actuators nano- and microdisplacement. International Journal of Mathematical Analysis and Applications 3(4): 31-38.

22. Afonin SM (2018) Structural-parametric model of electromagnetoelastic actuator for nanomechanics. Actuators 7(1): 1-9.
23. Afonin SM. (2019) Structural-parametric model and diagram of a multilayer electromagnetoelastic actuator for nanomechanics. Actuators 8(3): 1-14.

24. Afonin SM. (2016) Structural-parametric models and transfer functions of electromagnetoelastic actuators nano- and microdisplacement for mechatronic systems. International Journal of Theoretical and Applied Mathematics 2(2): 52-59.

25. Afonin SM. (2018) Structural-parametric model of electro elastic actuator for nanotechnology and biotechnology. Journal of Pharmacy and Pharmaceutics 5(1): 8-12.

26. Afonin SM. (2010) Design static and dynamic characteristics of a piezoelectric nanomicrotransducers. Mechanics of Solids 45(1): 123-132.

27. Afonin SM (2018) Electromagnetoelastic Actuator for Nanomechanics. Global Journal of Research in Engineering: A Mechanical and Mechanics Engineering 18(2): 19-23.

28. Afonin SM. (2018) Multilayer electromagnetoelastic actuator for robotics systems of nanotechnology, Proceedings of the 2018 IEEE Conference EIConRus, 1698-1701.

29. Afonin SM. (2018) A block diagram of electromagnetoelastic actuator nanodisplacement for communications systems. Transactions on Networks and Communications 6(3): 1-9.

30. Afonin SM. (2019) Decision matrix equation and block diagram of multilayer electromagnetoelastic actuator micro and nanodisplacement for communications systems, Transactions on Nnetworks and Communications 7(3): 11-21.

31. Afonin SM. (2020) Condition absolute stability control system of electromagnetoelastic actuator for communication equipment. Transactions on Networks and Communications 8(1): 8-15.

32. Afonin SM. (2020) A Block diagram of electromagnetoelastic actuator for control systems in nanoscience and nanotechnology, Transactions on Machine Learning and Artificial Intelligence 8(4): 23-33.

33. Afonin SM. (2020) Optimal control of a multilayer electroelastic engine with a longitudinal piezoeffect for nanomechatronics systems. Applied System Innovation 3(4): 1-7.

34. Afonin SM. (2020) Structural scheme actuator for nano research. COJ Reviews and Research 2(5): 1-3.

35. Afonin SM. (2018) Structural-parametric model electroelastic actuator nano- and microdisplacement of mechatronics systems for nanotechnology and ecology research. MOJ Ecology and Environmental Sciences 3(5): 306-309.

36. Afonin SM. (2019) Condition absolute stability of control system with electro elastic actuator for nano bioengineering and microsurgery. Surgery \& Case Studies Open Access Jjournal 3(3): 307-309.

37. Afonin SM. (2019) Electro elastic actuator for micro and nano surgical repairs. Open Access Journal of Biomedical Engineering and Biosciences 3(2): 270-272.

38. Afonin SM (2020) Multilayer engine for microsurgery and nano biomedicine. Surgery \& Case Studies Open Access Jjournal 4(4): 423-425.

39. Afonin SM. (2020) Structural-parametric model actuator of adaptive optics for composite telescope and astrophysics equipment. Physics \& Astronomy International Journal 4(1): 1821.

40. Nalwa HS. (2004) Encyclopedia of Nanoscience and Nanotechnology. Los Angeles: American Scientific Publishers. 10 Volumes. 